\title{
A DATA-REDUCTION METHOD FOR INTRALAMINAR COHESIVE PROPERTIES USING A BAYESIAN APPROACH
}

\author{
C. González ${ }^{1,2 *}$ \\ ${ }^{1}$ IMDEA Materials, C/Eric Kandel 2, 28906 - Getafe, Madrid, Spain \\ 2 Department of Materials Science, Polytechnic University of Madrid, E.T.S. de Ingenieros de \\ Caminos, 28040 Madrid, Spain \\ * carlosdaniel.gonzalez@imdea.org
}

The proposed method is based on the concurrent participation of two main ingredients. Firstly, a Bayesian engine necessary to sample the posterior distribution of the intralaminar cohesive parameters $\Theta=\left[\sigma_{c}, G_{c}\right.$, shape, $\left.\ldots\right]$ given a set of experimental observations $Y$ obtained from the intralaminar fracture tests. Normally, such observations corresponded to load displacement $P-$ $\delta$ and crack-displacement $\Delta a-\delta$ curves. Secondly, a surrogate model based on cohesive FEM simulations enabling the fast computing of the compact tension specimen response mandatory for the likelihood determination during the Bayesian inference process.
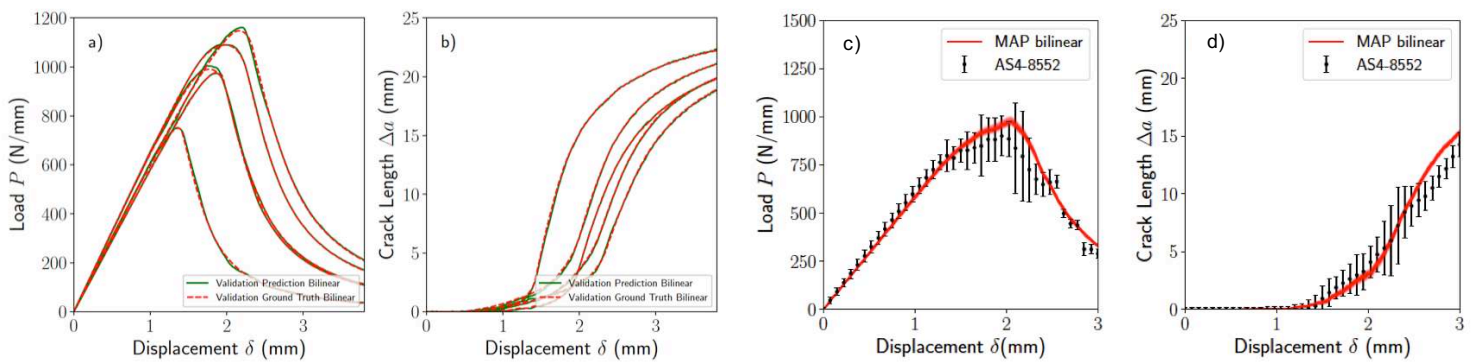

Figure 1: a,b) Comparison between ground truth and the predictions of the ANN surrogate model of the $P-\delta$ and crack-displacement $\Delta a-\delta$ curves given a set of arbitrary cohesive parameters, c,d) Comparison between experimental response and Bayesian inference quantification.

To this end, complete datasets of compact tension CT fracture tests using linear and bilinear cohesive laws were generated for the set of variables proposed (e.g. strength, toughness and softening curve shape). Virtual fracture tests were conducted sequentially using Abaqus Standard for each of the cohesive parameter combinations provided by the Latin Hypercube method. After completion, the datasets formed by 400 simulations were serialized into a Python pickle providing an easy access for the subsequent neural network training tasks. The ANN was coded in Keras being the surrogate produced able to predict the $P-\delta$ and crack-displacement $\Delta a-\delta$ curves given the set of cohesive parameters with errors less than $1 \%$ in the validation datasets (Figure $1 \mathrm{a}$ and $\mathrm{b}$ ). Such efficient surrogate overcomes the stringent requirements of fast computation required during Bayesian sampling. According to the Bayes theorem, the posterior probability $p(\Theta \mid Y)$, also known as the probability of the cohesive parameters $\Theta$ given the experimental observations $Y$, is given by $p(\Theta \mid Y)=p(Y \mid \Theta) p(\Theta) / p(Y)$ where $p(Y \mid \Theta)$ is the likelihood Gaussian function and $p(\Theta)$ is the prior distribution, while $p(Y)$ is known as the evidence of the experimental results. Posterior distributions $p(\Theta \mid Y)$ were sampled in this work by using the EMCEE Python implementation of the affine invariant Markov chain Monte Carlo (MCMC) Ensemble sampler. The results of the Bayesian approach were applied to ascertain the cohesive parameters from the CT experiments for AS4/8552 cross-ply laminates (Figure $1 \mathrm{c}$ and d). 